http://dx.doi.org/10.32911/as.2017.v10.n2.167

Aporte Santiaguino. 10 (2), 2017: 245-258

ISSN 2070-836X

\title{
Caracterización de fincas productoras de tuna (Opuntia ficus indica) para la producción de cochinilla del carmín (Dactylopius coccus) en La Joya (Arequipa, Perú)
}

\author{
Characterization of tuna farms (Opuntia ficus indica) for the production of \\ carmine mealybug (Dactylopius coccus) in La Joya (Arequipa, Perú) \\ aLbeRTo ancuLLe aRenas ${ }^{1}$, ViViana CasTRo CePeRo ${ }^{1}$ y aLbeRTo JuLca OTiniano ${ }^{1}$
}

\section{RESUMEN}

La investigación se realizó, en el distrito de La Joya (Arequipa, Perú), con el objetivo de caracterizar las fincas productoras de tuna para la producción de cochinilla del carmín. Se trabajó en las irrigaciones La Joya Antigua, El Triunfo, La Cano y San Isidro, tomando una muestra $(n=252)$ de una población total de 819 agricultores. Se aplicó un cuestionario de preguntas sobre los aspectos técnicos, sociales y económicos. Se halló que las fincas son complejas y diversas. La producción de cochinilla del carmín es una actividad muy importante, complementada con la ganadería lechera y cultivos (anuales o frutales) para el mercado local y de exportación. Los rendimientos e ingresos no son bajos, pero podrían ser mayores si se mejora la asociatividad y algunas técnicas de cultivo. Por la cercanía a la ciudad de Arequipa, se dispone de la mayoría de servicios básicos, aunque el servicio de agua potable y el asfaltado de vías internas, es deficiente. El análisis de conglomerados por el Método de Ward y una distancia Euclidiana Cuadrada de 600, determina seis grupos de fincas. El tipo VI, es el más frecuente $(23 \%$ del total), en la Irrigación La Cano, con 4.6 hectáreas instaladas con tuna (de un total de $8 \mathrm{ha}$ ), $574 \mathrm{~kg} \mathrm{ha}^{-1}$ de cochinilla seca y USD \$ 7418 de ingreso neto, por hectárea año, respectivamente.

Palabras clave: fincas; caracterización; tuna; cochinilla del carmín.

\begin{abstract}
The research was carried out in the district of La Joya (Arequipa, Peru) with the objective of characterizing tuna farms for the production of carmine mealybugs. Work was carried out on the La Joya Antigua, El Triunfo, La Cano and San Isidro irrigation
\end{abstract}

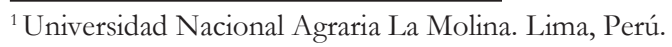


systems, taking a sample $(\mathrm{n}=252)$ from a total population of 819 farmers. A questionnaire of questions on technical, social and economic aspects was applied. It was found that the farms are complex and diverse. The production of carmine mealybugs is a very important activity, complemented by dairy farming and crops (annual or fruit) for the local and export market. Yields and incomes are not low, but they could be higher if the associativity and some cultivation techniques are improved. Due to the proximity to the city of Arequipa, most basic services are available, although the drinking water service and the asphalting of internal roads is deficient. The cluster analysis by the Ward Method and a Square Euclidean distance of 600, determines six groups of farms. Type VI is the most frequent (23\% of the total), in La Cano irrigation, with 4.6 hectares installed with tuna (of $8 \mathrm{ha}$ ), $574 \mathrm{~kg}$ ha-1 of dried cochineal and USD \$ 7418 USD net income, per hectare per year, respectively.

Keywords: farms; characterization; tuna; carmine mealybug.

\section{INTRODUCCIÓN}

La tuna (Opuntia ficus indica) es una especie perteneciente a la familia de las Cactaceae. Esta familia, se compone de tres subfamilias: Pereskioideae, con hojas llanas y suculentas, espinas y flores en panículas; Opuntioideae, con ramas planas y suculentas, hojas cilíndricas, suculentas y temporales, areolas con gloquidios y flores alternadas y Cereoideae (=Cactoideae), con tallos suculentos, hojas reducidas a espinas, areolas sin gloquidios y flores con forma de embudo. La subfamilia Opuntioideae está conformada por plantas arborescentes, arbustivas o cespitosas; tallos usualmente segmentados; zona fértil no diferenciada; hojas permanentes o temporales, pequeñas, terete; areolas con espinas tubuladas, aciculadas, con gloquidios; flores laterales, raramente terminales, sésiles, solitarias, diurnas; pericarpelo rodeado por una porción de tallo con areolas y espinas, hojas pequeñas; tubo del perianto corto o ausente, estambres numerosos, ovario ínfero; frutos baya con o sin pulpa; semilla circular, arilo rígido formado a partir del funículo. Está organizada en 5 tribus y 15 géneros (Anderson, 2001; Barthlott \& Hunt, 1993; Castro, 2006).

Plantas de O. ficus indica, se encuentra de manera espontánea en diferentes lugares de la sierra peruana; pero es en el departamento de Arequipa, donde probablemente su cultivo ha tenido mayor éxito. En el distrito de La Joya, ubicado en la sierra media arequipeña (1286 m s.n.m.), la tuna se cultiva para la producción de cochinilla del carmín (Dactylopius coccus), la que es exportada a diferentes países del mundo para su utilización como colorante natural por la industria alimenticia, farmacéutica y de cosméticos. Según la Asociación de Exportadores del Perú (Asociación de Exportadores, 2017), el Perú produce el $90 \%$ de la cochinilla del mundo y la región Arequipa concentra el $70 \%$ de la producción nacional, siendo el $60 \%$ proveniente del distrito de La Joya. En La Joya se tienen instaladas 3855 ha de tuna para cochinilla (gobierno regional de 
Arequipa, 2017), que produce 1 968,66 t de cochinilla del carmín seca por año, lo que hace a este distrito el principal productor del Perú. La producción de la cochinilla del carmín tiene importancia ambiental, social y económica en Arequipa y en La Joya, pues contribuye a generar ocupación, ingresos y oportunidades de desarrollo para trabajadores y productores. Sin embargo, como cultivo de reciente introducción a La Joya (aproximadamente 18 años), la complejidad y diversidad propia de las fincas productoras y la escasa información sobre las características de las mismas hace necesario un estudio que permita identificar los problemas más importantes. Esta información es necesaria para proponer alternativas de mejora, considerando los criterios de la sustentabilidad agrícola. El adecuado conocimiento del contexto del pequeño productor es la base para la investigación y transferencia de tecnología, teniendo en cuenta que esta debe generarse de acuerdo a ese contexto; además que la tipificación y clasificación de sistemas de fincas debe hacerse basada en un marco teórico específico para la tipificación y clasificación, selección de variables del sistema de finca, recolección de datos por medio de encuestas y otras formas, análisis multivariado de los datos obtenidos, validación de la tipología y clasificación de las fincas (Escobar y Berdegue, 1990). Es el caso del trabajo de García et al. (2010), quienes utilizaron el análisis multivariado para establecer tres tipos de sistemas ganaderos en las dehesas andaluzas. La caracterización de las fincas, se realiza considerando aspectos comunitarios y técnicos, y con los actores para establecer la situación actual de los recursos físicos y humanos como la población, educación, salud, organización, que permitan visualizar la situación a futuro (UICN, 2009). Permite también analizar y proponer alternativas de solución apropiadas, y lograr una mayor sustentabilidad y un uso eficiente de las mismas; lo que implica ubicar, delimitar, identificar y describir todos los componentes que integran la finca para realizar un ordenamiento de la misma bajo criterios de sustentabilidad (Fernández, Brooks y Cordón, 2008). A nivel mundial, de un total de 19 indicadores utilizados para caracterizar las explotaciones agrícolas para mejorar la formulación de políticas, se halló que las más frecuentes fueron: dimensión económica de la explotación; superficie de la tierra o superficie cosechada; composición de la mano de obra; importancia de las actividades fuera de la explotación; orientación a la producción o la especialización agrícola; orientación o integración al mercado; y la auto-subsistencia (Saravia, Cimpoies y Ronzon, 2013). En un análisis de la actividad de pequeños agricultores de Arequipa se halló que sus índices de productividad son altos pero que el trabajo es más individual que asociado (Obando, 1996). En Piura y Junín se halló una relación muy alta entre la eficiencia técnico-económica con el nivel de ingreso de los pequeños agricultores, siendo el principal determinante, sin lugar a duda, el nivel de educación y conocimiento técnico del productor (Escobal, 2006). Álvarez, Riveiro y Marey (2008) han desarrollado una metodología que agrupa y caracteriza explotaciones lecheras en tipologías de acuerdo con sus variables básicas: aprovechamientos que las integran, rangos dimensionales y sistemas de producción, basados en datos de los censos; y la caracterización y clasificación se hizo considerando el criterio de área cultivada para 
autoconsumo y para venta, producción de leche y uso de fertilizantes. Estudios de caracterización en cultivos perennes como café (Manabí, Ecuador), muestran que el 81 \% de los responsables de las fincas son varones; el $47 \%$ tiene una edad entre 41 y 60 años, y el $29 \%$ más de 60 años; el $57 \%$ solo tiene instrucción primaria y un $20 \%$ estudios superiores; un $47 \%$ usa como medios de información la televisión, folletos; tan solo el $8 \%$ de las casas son de hormigón y la mayor parte de son de madera $(28 \%$ y caña $(24$ $\%)$; y la mayoría de los encuestados (68\%) solo tiene luz y agua de pozo y un grupo muy pequeño posee los servicios luz, agua de pozo, desagüe y teléfono (Santistevan et al., 2014). Para fincas cacaoteras en San Martín (Perú), Tuesta et al. (2014), hallaron que la edad de los agricultores oscilaba entre 44 y 46 años, de 3 a 4 integrantes de la familia y que además de cacao desarrollaban cultivos anuales y ganadería, finalmente una gran proporción de fincas pertenecen a una organización, especialmente cooperativas. En otro caso de fincas limoneras (Santa Elena, Ecuador) se halló que los responsables de la finca son varones en un $95 \%$; el $67 \%$ tienen edades entre 46 - 65 años; el $77 \%$ solo tiene instrucción primaria y apenas un $2 \%$ de instrucción superior; el $71 \%$ posee casa de hormigón; el $64 \%$ posee luz, agua potable y teléfono celular; un $57 \%$ utiliza medios de información como la televisión, la radio y los folletos; finalmente un $70 \%$ pertenece a alguna asociación y de los cuales un $40 \%$ es una organización de productores (Santistevan, Julca y Helfgott, 2015).

El distrito de La Joya tiene un clima favorable que confiere ventajas comparativas para el cultivo de la tuna y la producción de la cochinilla durante todo el año, y permite que el contenido de ácido carmínico sea alto. Aquí los medianos productores de cochinilla del carmín también cultivan papa, cebolla, alfalfa y maíz forrajero; pero esta diversidad de cultivos puede variar por la tenencia de la tierra, el tamaño de la unidad productiva, la organización de la producción, monto de la inversión empleada, forma de contratación de la mano de obra y forma de comercialización (Valdivia, 2006). Este trabajo se realizó con el objetivo de caracterizar las fincas productoras de tuna (Opuntia ficus indica) para la producción de cochinilla del carmín (Dactylopius coccus) en La Joya (Arequipa, Perú).

\section{MATERIALES Y MÉTODOS}

El estudio se realizó en el distrito de La Joya (figura 1), provincia y región Arequipa, ubicado al oeste de la ciudad de Arequipa (a $60 \mathrm{~km}$, aproximadamente). La altitud varía de 1.169 y 1.665 m s.n.m. (en promedio 1.286); la superficie total es de $670,22 \mathrm{~km}^{2} \mathrm{y}$ se ubica a $16^{\circ} 43^{\prime} 34^{\prime \prime} \mathrm{S}$ y $71^{\circ} 51^{\prime} 40^{\prime \prime}$ W. La temperatura media anual es de $18,5^{\circ} \mathrm{C}$, la mínima media de $9,8^{\circ} \mathrm{C}$ y la máxima media de $26,5^{\circ} \mathrm{C}$. La humedad relativa es $29,5 \mathrm{y}$ 20,1\%, en el aire y suelo, respectivamente. Los vientos son alisios, de día soplan de sur a norte y de noche de norte a sur. La radiación solar media anual es de $508 \mathrm{~W} \mathrm{~m}^{-2}$ con una máxima de $1060 \mathrm{~W} \mathrm{~m}^{-2}$. 


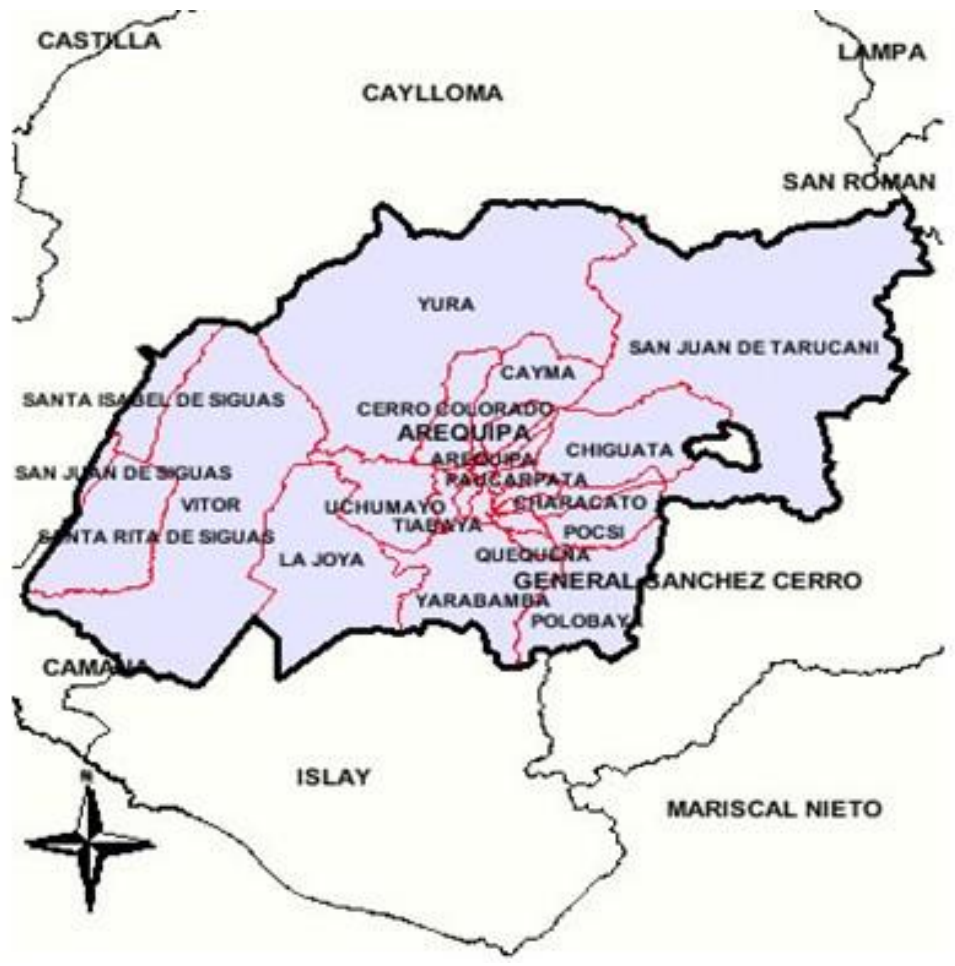

Figura 1. Mapa político del distrito de La Joya, en la provincia de Arequipa (Perú), marcado con un círculo como la zona de estudio

La Joya, es parte de la cuenca de los ríos Chili-Quilca y se encuentra en la zona de vida desierto subtropical; el clima es extremadamente árido y semicálido; los suelos agrícolas son de origen aluvial y están ubicados en la zona plana de la llanura aluvial, con un relieve topográfico de ondulado a plano, pendiente de 2 a $4 \%$ (dirección este-oeste), contenido de materia orgánica mínimo a medio, textura arena franca a franco arenoso, sin problemas de drenaje y salinidad, y permeabilidad moderada a rápida; precipitaciones muy escasas (promedio anual $1.8 \mathrm{~mm}$ ); y finalmente el área cultivada es de 10193 ha (67 y $35 \%$ con cultivos permanentes y transitorios, respectivamente), de las cuales 8848 ha tienen riego (MINAGRI-ANA, 2008).

El clima de las zonas comprendidas entre los 0 y los 2000 m s.n.m, al no tener temperaturas inferiores a $6{ }^{\circ} \mathrm{C}$ ni mayores a $35^{\circ} \mathrm{C}$, asemeja a un gran invernadero en condiciones naturales (Jiménez et al., 2002), afirmación que concuerda con lo reportado por el MINAGRI-ANA (2008) quienes señalan que en esta zona, las condiciones climáticas uniformes durante el año y las fuentes de agua de un sistema de represas brindan ventajas comparativas naturales extraordinarias para la explotación de una gran variedad de cultivos, en las diferentes épocas del año.

En el área de estudio se ubican las irrigaciones La Joya Antigua, El Triunfo, La Cano, San Isidro y San Camilo, agrupadas en dos juntas de usuarios (La Joya Antigua y La 
Joya Nueva). Entre las dos Juntas de Usuarios, se registran una población de 819 productores de cochinilla del carmín, de la que se tomó una muestra $(n=252)$ usando la fórmula propuesta por INCAGRO (2006) y repartida entre los agricultores de La Joya Antigua (89), El Triunfo (20), La Cano (75) y San Isidro (68); no se consideró a San Camilo porque el área instalada con este cultivo es muy pequeña. La información de las fincas evaluadas se obtuvo mediante la aplicación de un cuestionario de preguntas relacionadas con los aspectos técnicos, sociales y económicos. Luego se realizó un análisis de conglomerados usando el Método de Ward, con una distancia Euclidiana Cuadrada de 600.

\section{RESULTADOS Y DISCUSIÓN}

Características del productor de tuna para cochinilla del carmín. En la figura 2 se observa que los productores de cochinilla del carmín que residen en la finca son el $87 \%$, el $12 \%$ en centros poblados cercanos a la finca y el $1 \%$ en la ciudad. La residencia en la finca permite una mayor acción directa y vigilancia sobre el proceso productivo y de comercialización. El INEI (2013) reporta que en el distrito de La Joya el 55 \% de los productores en general viven en las unidades agropecuarias, lo que es menor en un 32 \% comparado con los productores de cochinilla del carmín, tal vez porque en este caso la actividad requiere de mayor presencia en la finca, comparado con otros cultivos o crianzas. El 89 \% de las fincas productoras de cochinilla en La Joya (figura 3), son conducidas por los varones, lo que indica que en la mayor parte los hogares se encuentran constituidos. El $70 \%$ de los encuestados tiene una edad menor a 60 años (figura 4), edad que le permite al productor dedicarse a la actividad productiva plenamente y el 30 \% más de 60 años. Para la instrucción (figura 5) el 37, 20 y 43 \% tienen instrucción secundaria, solo primaria e instrucción superior, respectivamente, hecho que indica que existe un capital humano con conocimientos y preparación académica que es muy útil en el desarrollo de la actividad, y sobre todo porque del $43 \%$ que cuenta con instrucción superior, el $8 \%$ es de formación no universitaria, el $34 \%$ superior universitaria y el $1 \%$ con posgrado. El $44 \%$ de los agricultores productores de cochinilla del carmín tienen de 2 a 3 familiares dependientes y el $30 \%$, de 4 a 5 familiares. En estos aspectos el INEI (2013) reporta que el $68 \%$ de los agricultores responsables de las fincas son de sexo masculino, para la actividad del distrito, a diferencia del $89 \%$ dirigido por varones en el caso de la cochinilla del carmín; y que el $47 \%$ de los agricultores tienen entre 45 y 64 años de edad, lo que es consistente con lo hallado en el estudio; en la instrucción el $48 \%$ tiene instrucción secundaria, $21 \%$ solo primaria y $28 \%$ instrucción superior, lo cual es semejante para la instrucción primaria y secundaria, pero inferior a la instrucción superior encontrada en la investigación; el 49\% tiene de 2 a 3 integrantes por familia y el $21 \%$ de 4 a 5 integrantes, resultados cercanos a los hallados en el estudio. Las características del responsable y residencia del mismo en la finca así como la edad, el grado de instrucción y los integrantes de la familia hallado en el estudio resultan fa- 
vorables para la búsqueda de la sustentabilidad de las fincas productoras de tuna para cochinilla del carmín en el distrito de la Joya, en Arequipa.

La utilidad por productor de cochinilla, por hectárea año (figura 6), para el momento de evaluación y en el que el precio del kilogramo de cochinilla seca era de USD \$ 40.00, muestra que el $58 \%$ tiene un ingreso neto de más de USD \$ 7500 y un $25 \%$ entre US\$ 5625 y USD \$ 7500 ; así el $83 \%$ de productores tiene un ingreso neto de más de US\$ 5 625. Estos valores muestran los altos ingresos por hectárea y por productor.

Las fincas evaluadas cuentan con servicio de transporte público, vías carrozables y las principales vías están asfaltadas. De los productores encuestados, el $92 \%$ poseen casa propia y para el tipo de construcción un $44 \%$ posee una vivienda construida completamente con ladrillo y cemento, y acabados; mientras que un $42 \%$ posee una vivienda construida completamente con ladrillo y cemento, y sin acabados; y solo un $3 \%$ posee una vivienda construida con adobe, calamina o esteras de carrizo; las construcciones de material noble evidencian los efectos de la cochinilla del carmín, sobre todo por los precios altos de años anteriores. El $62 \%$ compran agua potable de tanques cisterna y el $86 \%$ no disponen de desagüe. El $99 \%$ disponen de luz eléctrica. El 99\% usa teléfono, de los cuales $64 \%$ es de tipo celular. El $61 \%$ no dispone de internet y el $35 \%$ sí posee, pero inalámbrica.

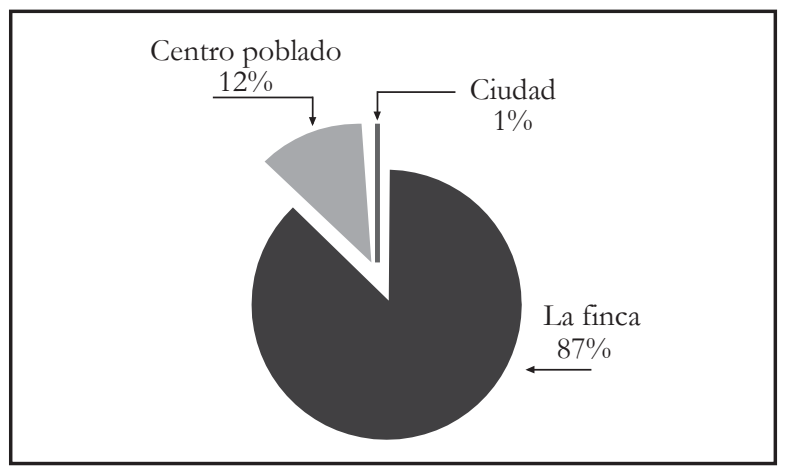

Figura 2. Lugar de residencia de los productores de cochinilla del carmín

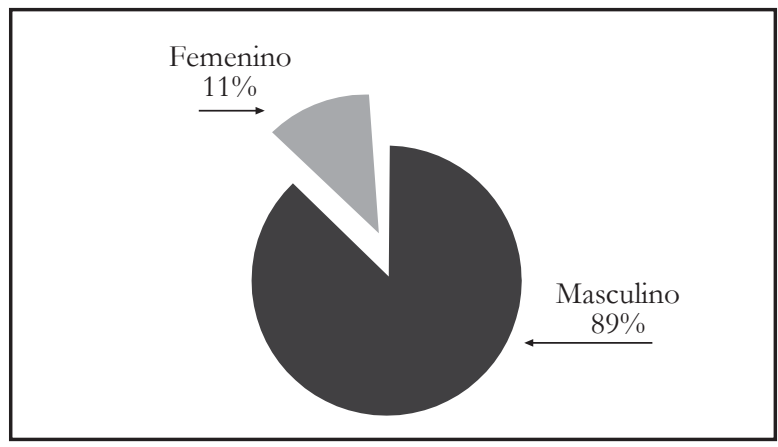

Figura 3. Género del responsable de la finca productora de cochinilla del carmín 


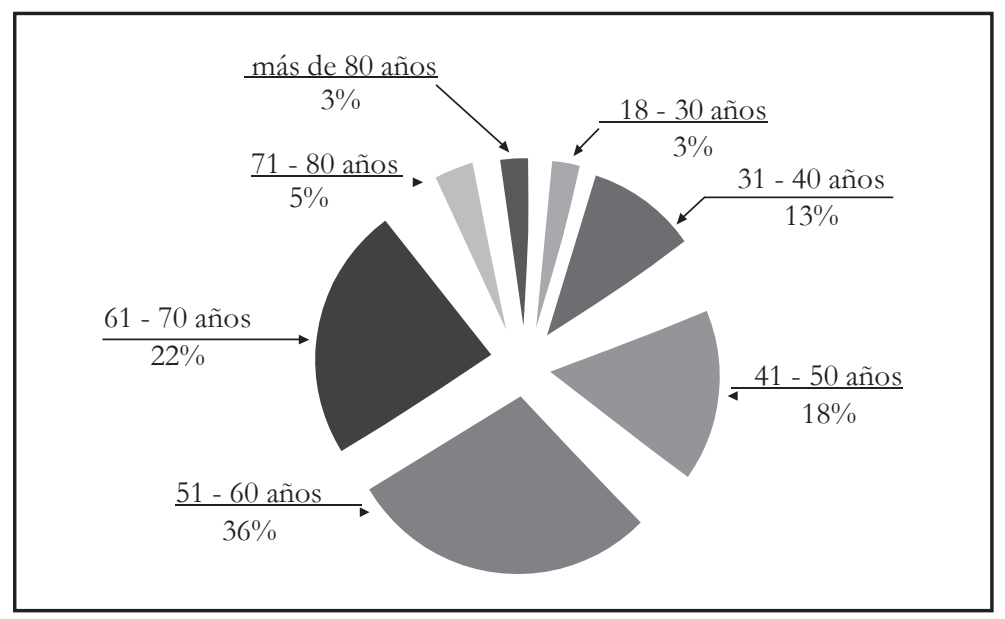

Figura 4. Edad del responsable de la finca productora de cochinilla del carmín

El $60 \%$ dispone de televisión normal y el 40 \% por cable. Finalmente, para la atención de su salud el $65 \%$ recurre a hospitales nacionales y el $31 \%$ a servicios particulares. La mayor deficiencia de los servicios básicos para los productores es la falta de agua potable de tubería y los servicios de internet alámbrica, que fomente la comunicación y búsqueda de información. Solo el $23 \%$ se dedica a la producción de cochinilla del carmín (figura 7) y el $77 \%$ se dedica además a otras actividades como producción de otros cultivos (43\%), ganadería lechera (24\%), trabajador agrícola (6\%), como profesional $(3 \%)$ y como comerciante $(1 \%)$; la experiencia del productor sobre los precios de la cochinilla del carmín, que pueden llegar a estar por debajo del costo de producción, explica por qué el productor busca seguridad y diversifica sus ingresos con otras actividades agrícolas.

Actividades productivas. Según lo manifestado por los productores de la cochinilla del carmín, los campos de tuna están instalados con una mezcla de los cultivares 'Amarilla', 'Morada' y 'Blanca'; la 'Morada' representa casi el $95 \%$ de la población en los campos, la 'Amarilla el $5 \%$ y la 'Blanca' menos del $1 \%$. El tamaño promedio de las fincas (figura 8 ) es de un poco más de 5 ha, con $55 \%$ que tiene menos de 3 ha y un $26 \%$ de 4 a 6 ha, lo que suma un $81 \%$ con menos de 6 ha. y un $19 \%$ con más de 7 ha; esto se explica en parte por el diseño inicial de las irrigaciones que fueron planificadas con tamaños de parcela de 5, 7 o 10 ha. La Joya tiene cerca del $32 \%$ de agricultores con propiedades menores a 6 ha y cerca del $53 \%$ menos de 10 ha; por su parte el MINAGRI - ANA (2008) señalan que hay predominio de la mediana propiedad y que el 77 $\%$ de los productores tiene en propiedad entre 1 a 10 ha. El área pequeña que posee el agricultor de La Joya explica porque las áreas destinadas al cultivo de tuna para cochinilla sean también pequeñas. El Gobierno Regional de Arequipa (2017) reporta, para La Joya, un área instalada de tuna para cochinilla del carmín de 3855 ha para la campaña 
2014 - 2015. Por otro lado, las juntas de usuarios de La Joya Nueva y La Joya Antigua registran un área promedio de cerca de 6 ha por productor de cochinilla del carmín, lo cual es consistente a lo encontrado en este estudio.

Se puede observar que los rendimientos de cochinilla del carmín seca en La Joya varían de 200 a más de $800 \mathrm{~kg} \mathrm{ha}^{-1}$, estando el $44 \%$ de fincas con rendimientos entre $500 \mathrm{y}$ $600 \mathrm{~kg} \mathrm{ha}^{-1}$. Estos rendimientos se asemejan a los reportados por el Gobierno Regional de Arequipa (2017) para las cosechas del año 2012, 2013 y 2014 con promedios de 688, 633 y $314 \mathrm{~kg} \mathrm{ha}^{-1}$ de cochinilla seca, respectivamente; sin embargo para la campaña 2013-2014 bajó a 314 kg ha-1, explicado por la disminución de los precios de venta. Los precios promedio de venta anual, por kilogramo de cochinilla seca en chacra, estimados por el Gobierno Regional de Arequipa (2017), varían desde USD \$ 20,7; 17,4; 12,4; 18,5; 59,6 y 82,0; para las cosechas de los años 2015, 2014, 2013, 2012, 2011 y 2010 respectivamente.

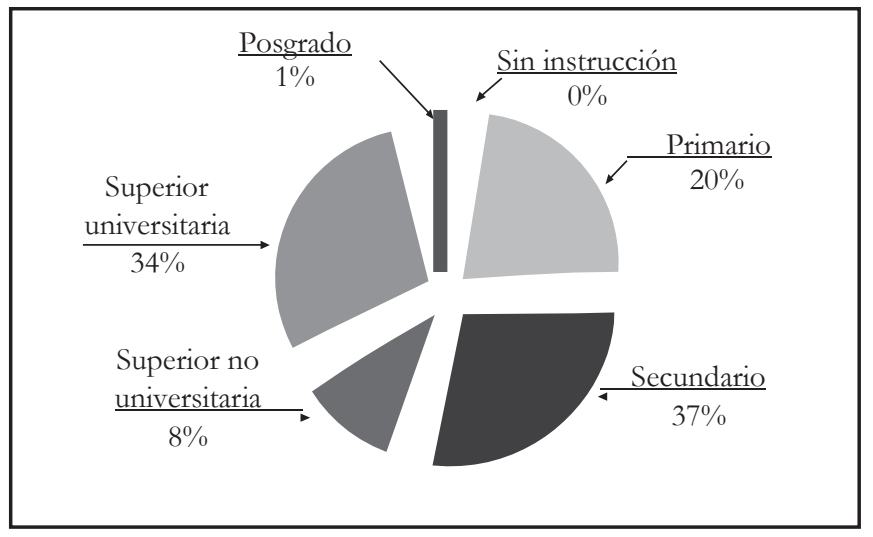

Figura 5. Grado de instrucción del responsable de la finca productora de cochinilla del carmín

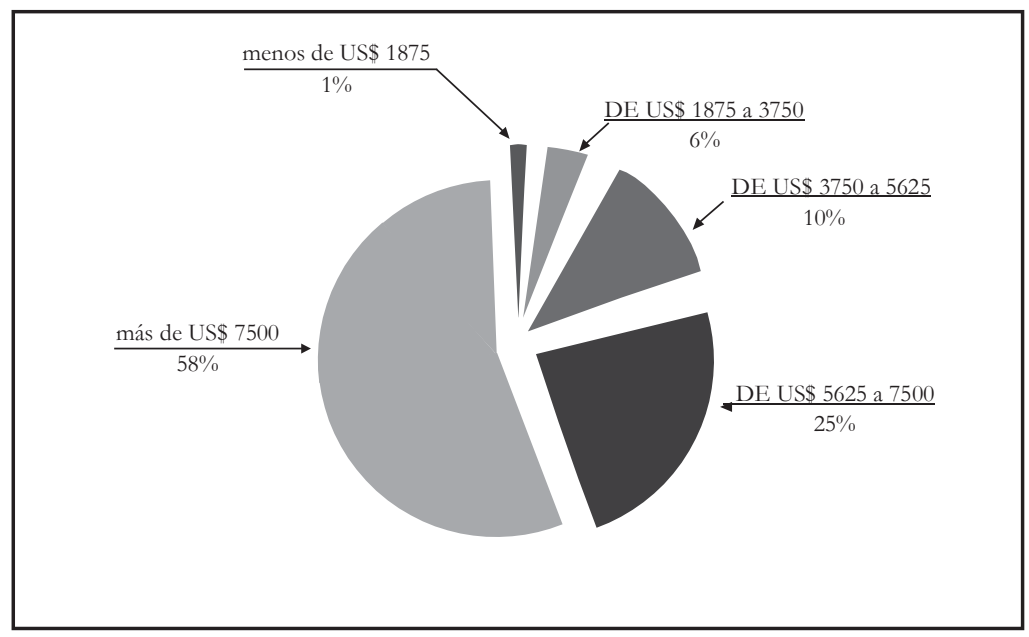

Figura 6. Ingresos netos (USD \$/hectárea/año) de los productores de cochinilla del carmín 


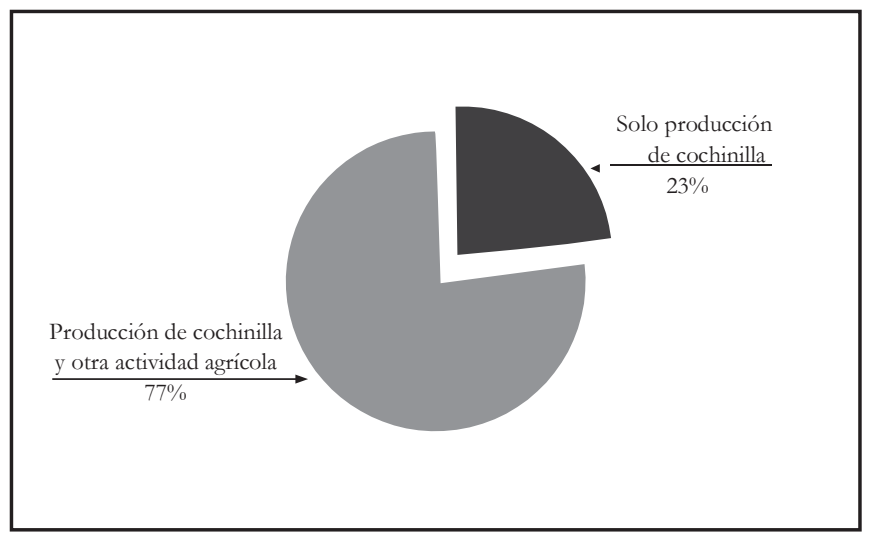

Figura 7. Actividades realizadas por los productores de cochinilla del carmín

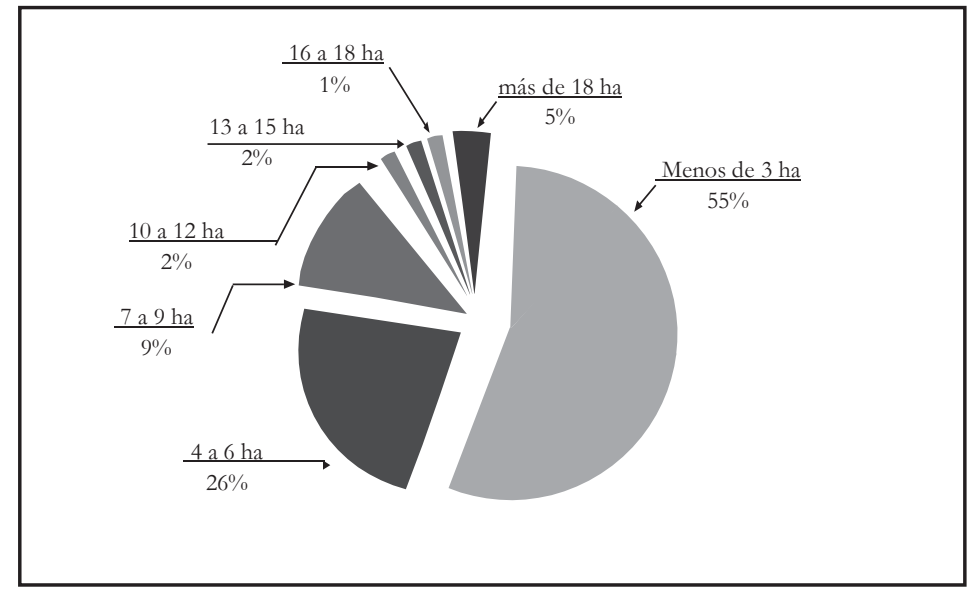

Figura 8. Área instalada de tuna para la producción de cochinilla del carmín

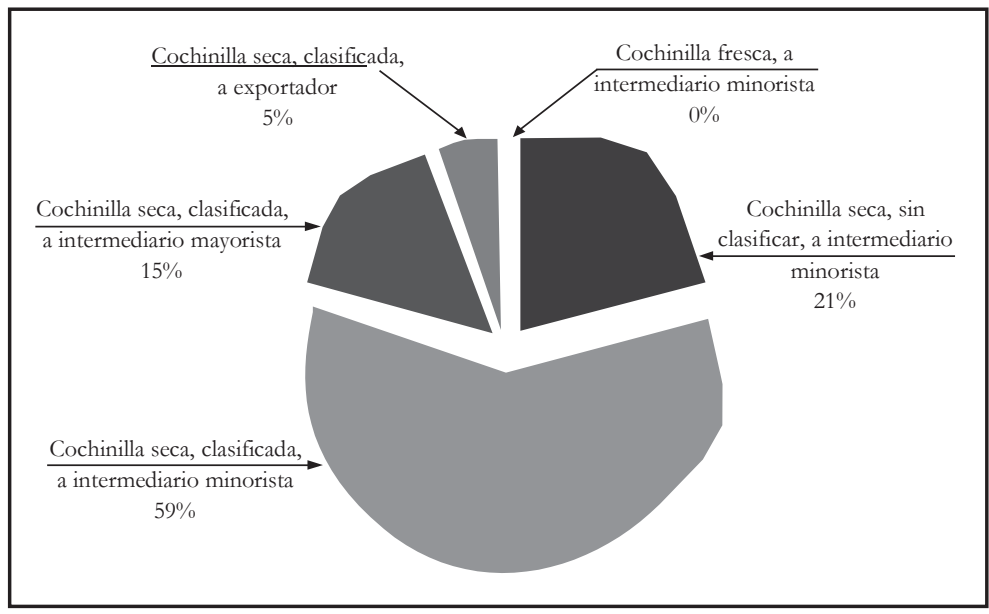

Figura 9. Forma de comercialización de la cochinilla del carmín 


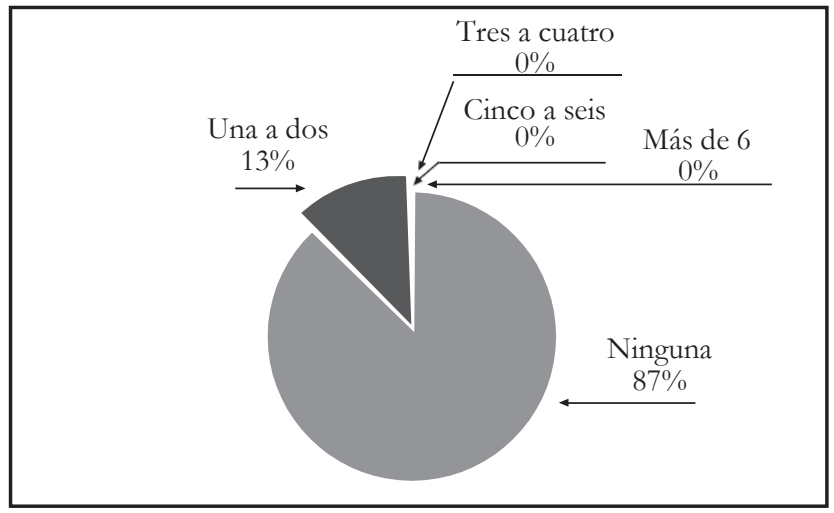

Figura 10. Número de asociaciones a las que pertenece el productor que produce y comercializa cochinilla del carmín

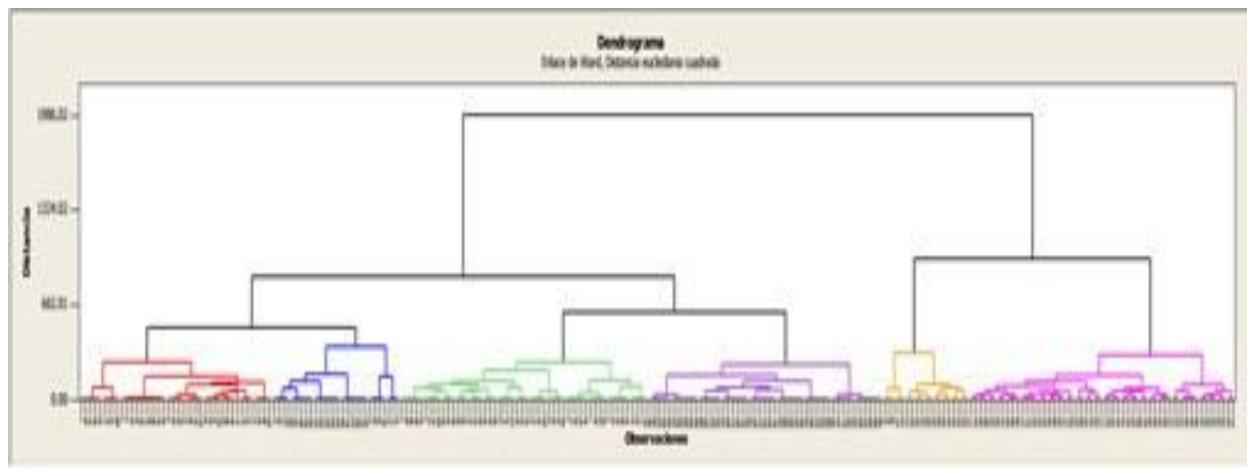

Figura 11. Agrupamiento de fincas productoras de tuna para la producción de cochinilla del carmín en La Joya (Arequipa-Perú) con el método de Ward y una distancia Euclidiana Cuadrada de 600. Los seis grupos se presentan con colores diferentes

Como se indicó anteriormente en el momento que se realizó el estudio (diciembre de 2015-enero de 2016) el precio fue de USD 40.00. El 59 \% de los productores vende la cochinilla seca y clasificada a un intermediario minorista (figura 9), mientras el $21 \%$ vende la cochinilla seca y sin clasificar a intermediario minorista, el $15 \%$ vende cochinilla seca a intermediario mayorista y tan solo el $5 \%$ vende directamente al exportador. El $53 \%$ de productores de tuna para cochinilla del carmín, instalan además cultivos como ajo, cebolla, papa, maíz chala, alfalfa, vid y palto. El $82 \%$ de los agricultores solo instala tuna sin otro cultivo asociado y el $15 \%$ puede instalar otro cultivo entre las columnas de tuna y generalmente este es maíz. La producción de cochinilla del carmín no permite la rotación de cultivos en forma inmediata como ocurre con los cultivos anuales, puesto que es un cultivo perenne y después de 5 a 10 años, en promedio, se hace rotación con cultivos como, ajo, papa, maíz chala o alfalfa. El Gobierno Regional de Arequipa (2017) señala para La Joya cultivos como zanahoria, vid, tara, quinua, poro, papa, paprika, palto, pacae, tuna para cochinilla, manzano, higuera, granado, maíz 
chala, chía, cebolla, alfalfa, alcachofa y ajo. La asociatividad para la producción y comercialización de la cochinilla del carmín es muy baja (figura 10), puesto que se halló que tan solo el $13 \%$ se encuentra asociado mientras que el $87 \%$ no lo está. Un aspecto importante del cultivo de tuna para la producción de la cochinilla del carmín es el hecho de que se está haciendo uso de la biodiversidad con fines comerciales y de exportación; considerando que la tuna ha sido un recuso genético subutilizado, considerada como especie cultivada pero no domesticada; además de sus bajos requerimientos de agua y nutrientes del suelo, y la baja incidencia de plagas.

El análisis de conglomerado por el Método de Ward y con una distancia Euclidiana Cuadrada de 600 (figura 11), agrupa las fincas en seis grupos o tipos de fincas, como se puede observar en la tabla 1.

Tabla 1. Características más importantes de las "fincas tipo" que producen tuna para la producción de cochinilla del carmín en La Joya, Arequipa, Perú

\begin{tabular}{|c|c|c|c|c|c|c|}
\hline \multirow{2}{*}{ CARACTERÍSTICAS } & \multicolumn{6}{|c|}{ FINCA TIPO DE CADA GRUPO } \\
\hline & I & II & III & IV & $\mathrm{V}$ & VI \\
\hline Cantidad que representa (\%) & 16 & 11 & 21 & 21 & 8 & 23 \\
\hline Área promedio con tuna (ha) & 4.4 & 2.6 & 2.8 & 2.1 & 16.0 & 4.6 \\
\hline Área promedio total (ha) & 10.9 & 6.2 & 4.6 & 5.2 & 20.0 & 8.0 \\
\hline $\begin{array}{l}\text { Rendimiento cochinilla seca } \\
\qquad\left(\mathrm{kg} \cdot \mathrm{ha}^{-1}\right)\end{array}$ & 563 & 611 & 612 & 477 & 589 & 574 \\
\hline Ingreso neto (USD/ha/año) & 7250 & 6910 & 7703 & 5121 & 7813 & 7418 \\
\hline Irrigación & $\begin{array}{l}\text { La Joya } \\
\text { Antigua }\end{array}$ & $\begin{array}{l}\text { La Joya } \\
\text { Antigua, } \\
\text { San Isidro }\end{array}$ & $\begin{array}{l}\text { La Joya } \\
\text { Antigua, } \\
\text { El Triunfo }\end{array}$ & $\begin{array}{l}\text { San } \\
\text { Isidro }\end{array}$ & $\begin{array}{c}\mathrm{La} \\
\text { Cano }\end{array}$ & La Cano \\
\hline
\end{tabular}

El tipo de finca más frecuente es la del grupo VI, que se ubica en la irrigación La Cano, representa el $23 \%$ del total de fincas, con 4.6 hectáreas instaladas con tuna (de un total de 8); el rendimiento promedio año de cochinilla seca es de $574 \mathrm{~kg} \mathrm{ha}^{-1}$, un ingreso neto promedio por hectárea de USD 7418. 171. La finca menos frecuente es la del grupo V, corresponde solamente al $8 \%$ del total, tiene 16 ha sembrada con tuna (de 20 ha) y sus ingresos son de USD 7813 anuales. Las fincas tipo III y IV, tienen la misma cantidad (21\%), pero el rendimiento de cochinilla seca es más alto en la finca tipo III, lo que le permite tener un ingreso $50 \%$ superior al año.

\section{CONCLUSIONES}

Las fincas instaladas con tuna para la producción de cochinilla del carmín en el distrito de La Joya, muestran mucha diversidad y la producción de la cochinilla del carmín se ha convertido en una actividad muy importante, aunque hay otras actividades complementarias como la ganadería lechera y cultivos (anuales o frutales) para mercado 
local y de exportación. Los rendimientos y los ingresos, si bien no son bajos, podrían mejorarse con un mayor grado de asociatividad y algunas técnicas de cultivo más eficientes. La cercanía a la ciudad de Arequipa, permite que se disponga de la mayoría de los servicios básicos, aunque el agua potable y el asfaltado de las vías secundarias son aún deficientes y su mejora ayudaría a aumentar la calidad de vida del productor.

\section{AGRADECIMIENTOS}

Asociación de productores de cochinilla del carmín de La Cano, Junta de Usuarios La Joya Antigua, Junta de Usuarios La Joya Nueva.

\section{REFERENCIAS BIBLIOGRÁFICAS}

Asociación de exportadores. 2017. Fortalecimiento de la cadena de cochinilla. Arequipa-Perú.

Álvarez, Carlos; Riveiro, José y Marey, Manuel. 2008. Typology, classification and characterization of farms for agricultural production planning. Spanish Journal of Agricultural Research 6(1): 125-136.

Anderson, Edward. 2001. The Cactus Family. Timber Press, Inc. U.S.A. 776.

Barthlott Wilheim y Hunt David. 1993. «Cactaceae». En: K. Kubitzki (Ed) The families and genera of vascular plants. Vol II: Flowering Plants. Dicotyledons. Springer-Verlag Berlin Heidelberg. Alemania. 161-196.

Castro, Viviana. 2006. Taxonomía de la familia Cactaceae en el valle de río Chillón, Lima: cerro Umarcata y quebrada Orobel. Tesis para optar el Grado Académico de Magíster Scientiae en Ecología Aplicada. Universidad Nacional Agraria La Molina. 96 pág.

Escobar, Germán y Berdegue, Julio. 1990. «Conceptos y metodología para la tipificación de sistemas de finca: la experiencia de RIMISP». Páginas: 13-44. En: G. Escobar Germán y Julio Berdegue (eds.). Tipificación de sistemas de producción agrícola. Red Internacional de Metodología de Investigación de Sistemas de Producción (RIMISP). Santiago-Chile.

Escobal, Julio. 2006. «Cómo elevar la eficiencia y rentabilidad dela pequeña agricultura comercial». En: Trivelli, Carolina; Escobal, Julio y Bruno Revesz (eds.). Pequeña agricultura comercial: dinámica y retos en el Perú, CIES, CIPCA, GRADE e IEP.

Fernández, Eduardo; Brooks, Morlan y Cordón Enrique. 2008. Estudio biofísico de la finca académica Snaki - URACCAN, en la comunidad de Moss, municipio de Waspam Rio Coco, $\mathrm{R} A A N 21$. Ciencia e Interculturalidad, Edición $\mathrm{N}^{\circ}$ 2: 44-66.

García, A. y otros. 2010. Structural characterization of extensive farms in andalusiandehesas. Arch. Zootec. 59 (228): 577-588.

Gobierno Regional de Arequipa. 2017. Estadística agrícola. Gerencia Regional de Agricultura. 
INCAGRO. 2006. Proyecto de investigación y extensión agricola-PIE $A$. Estudios de línea de base $y$ de salida de subproyectos cofinanciados. INCAGRO (Innovación y Competitividad para el Agro Peruano)-Ministerio de Agricultura.

Instituto Nacional de Estadística e Informática (INEI). 2013. Resultados definitivos IV Censo Nacional Agropecuario 2012.

Jiménez, Percy y otros. 2002. «Ecosistemas de Arequipa. Oferta Ambiental y Desarrollo Sostenible». Revista Zonas Áridas. Vol. 7, N 1:118-132.

MINAGRI-ANA. 2008. Diagnóstico de problemas y conflictos en la gestión del agua en la cuenca Chili-Quilca. Ministerio de Agricultura. Autoridad Nacional del Agua (ANA). Proyecto de Modernización de la Gestión de los Recursos Hídricos (PMGRH). Lima-Perú.

Obando, Marcos. 1996. Modelo de conducción agrario en el V alle de Arequipa. Documento de Trabajo N ${ }^{\circ} 77$. No77 Serie Talleres No5. Instituto de Estudios Peruanos. Lima. Perú. 35.

Santistevan, Mercedes y otros. 2014. Caracterización de fincas cafetaleras en la localidad de Jipijapa (Manabi, Ecuador). Ecología Aplicada 13(2): 187-192.

Santistevan, Mercedes; Julca, Alberto y Helfgott, Salomón. 2015. «Caracterización de las fincas productorasdel cultivo limón en las localidades de Manglar Alto y Colonche (Santa Elena, Ecuador)». Revista Cientifica y Tecnológica UPSE. Vol. 3 (1): 133-142.

Saravia, Silvia; Cimpoies, Dragos y Ronzon, Tevécia. 2013. Typology and Indicators to Characterize Agricultural Holdings for Improved Policy Formulation. Overviewof typologies of agricultural holdings in different geographical areas and focus. GCP/GLO/462/IFA Methodologies and pilot studies for a World Agriculture Watch (WAW).

Tuesta, Oscar y otros. 2014. Tipología de fincas cacaoteras en la subcuenca media del río Huayabamba, Distrito de Huicungo (San Martín, Perú). Ecología Aplicada, 13(2): 71-78.

UICN. 2009. Guía para la elaboración de planes de manejo de microcuencas. Proyecto Tacaná. Unión Internacional para la Conservación de la Naturaleza -UICN. Guatemala.

Valdivia, Gustavo. 2006. Haz de cuenta que yo era hombre y que mantenía a mi mujer: globalización, género y trabajo asalariado en una zona de agroexportación.

Recibido: $20 / 07 / 17$

Aceptado: 26/10/17

\section{Correspondencia}

Alberto Anculle Arenas

albertoanculle@gmail.com 